\title{
Deadlocks in the synchronization of pulse-coupled oscillators on star graphs
}

\author{
Arke Vogell $\odot$, Udo Schilcher $\odot$, and Christian Bettstetter $\odot$ \\ Institute of Networked and Embedded Systems, University of Klagenfurt, Klagenfurt, Austria
}

(Received 5 June 2020; revised 23 October 2020; accepted 15 November 2020; published 14 December 2020)

\begin{abstract}
Some convergence proofs for systems of oscillators with inhibitory pulse coupling assume that all initial phases reside in one half of their domain. A violation of this assumption can trigger deadlocks that prevent synchronization. We analyze the conditions for such deadlocks in star graphs, characterizing the domain of initial states leading to deadlocks and deriving its fraction of the state space. The results show that convergence is feasible from a wider range of initial phases. The same type of deadlock occurs in random graphs.
\end{abstract}

DOI: 10.1103/PhysRevE.102.062211

\section{INTRODUCTION}

Pulse-coupled oscillators were introduced to model and explain synchronization phenomena in natural systems $[1,2]$. Each oscillator's phase variable $\phi \in[0,1]$ increases with time until it reaches the threshold $\phi=1$, where the oscillator "fires" to send a pulse to adjacent oscillators and to reset $\phi$ to zero. Whenever an oscillator receives a pulse, it adjusts its own phase according to a phase response function $h(\phi)$. In nature, the evolution of the phase and its reaction to receiving a pulse are predetermined (e.g., in neurons [3,4]). In human-made systems, however, they are often configurable or programmable features [5-7].

Ever since the dynamics and synchronization of pulsecoupled oscillators were analyzed rigorously [8], scientists have taken various approaches to proving and investigating the convergence to a synchronized state. This was done for different network types [8,9] and phase response functions [10-14]. Arbitrary networks do not necessarily synchronizeconvergence proofs exist only for certain assumptions or restrictions. For instance, it is known that globally coupled oscillators synchronize for almost all initial conditions with a phase response function that implements a constant phase jump in the positive direction (excitatory coupling) [8] or in the negative direction (inhibitory coupling) [10,15]. With some restrictions on the phase response function and temporal evolution of $\phi$, synchronization is shown numerically for lattices [11] and with almost no restriction for chains and directed trees [16]. In directed ring graphs, synchronization can be achieved through sufficiently strong coupling for arbitrary initial conditions [17]. Some systems do not converge for certain initial conditions. The probability of convergence to synchrony has been derived for aperiodic, strongly connected, directed graphs [18]. For arbitrary, connected graphs,

Published by the American Physical Society under the terms of the Creative Commons Attribution 4.0 International license. Further distribution of this work must maintain attribution to the author(s) and the published article's title, journal citation, and DOI. convergence proofs exist that restrict the initial phase for each oscillator to a half-arc in the phase space $[6,16]$. This restriction is motivated by some graphs prohibiting at least one node from ever firing if inhibitory coupling is involved. An example of a type of graph exhibiting this phenomenon is the star graph [6].

Many publications on pulse-coupled oscillators aim to show that certain types of graphs of pulse-coupled oscillators synchronize. In contrast to this, we study systems that do not synchronize. It is known that pulse-coupled oscillator systems may exhibit periodic states, also called phase-locked states in reference to the eponymous phenomenon in continuously coupled oscillators [19]. We empirically observe that several randomly initialized networks of pulse-coupled oscillators do not just evolve into periodic states but actually exhibit deadlocks. In a deadlock state, at least one node is permanently prevented from firing and thus never synchronizes with the rest of the network.

In this paper, we derive the entire domain of initial configurations prohibiting the firing of the center node in a star graph. The occurrence for such a deadlock was observed in our previous work [6], where it was circumvented by introducing the concept of stochastic coupling, in which a pulse is sent with a certain probability only. We now provide a more thorough analysis of this deadlock mechanism. Our insights pave the way to understanding the conditions that lead to a deadlock. In particular, we determine how likely it is for the center node in a randomly initialized star graph to be prevented from firing and show by simulation that deadlocks occur in random graphs as well.

We study star graphs for multiple reasons: First, the star graph is known to suffer from deadlocks [6]. Second, star graphs on their own are very relevant in real-world networks. To give an example, they play a prominent role in local area computer and telecommunication systems, which are often designed as a "star topology" with a central hub, base station, or access point. Third, large and complex networks often include hubs with many links to other nodes and thus contain stars as basic subgraphs. This generally includes small-world networks [20] and specifically power grids [21] and computer networks [22]. 


\section{MODEL}

A star graph consists of a single center node $v_{c}$ and $n$ leaf nodes $v_{1}, \ldots, v_{n}$ with links between $v_{c}$ and each leaf node but no links between the leaf nodes. All nodes are identical oscillators with an internal phase $\phi \in[0,1]$ increasing linearly with time. The oscillators interact through exchanging pulses via the links of the network. While no pulses are received and $\phi<1$, each phase evolves with time $t$ according to $d \phi / d t=1$. A pulse is sent to all adjacent nodes whenever $\phi=1$, at which point $\phi$ is also reset to zero. Upon receiving a pulse, an oscillator's phase is changed to $h(\phi)$, where $h:[0,1] \rightarrow[0,1]$ is the piecewise linear phase response function

$$
h(\phi)= \begin{cases}(1-a) \phi & \text { for } \phi \leqslant 0.5 \\ (1-a) \phi+a & \text { for } \phi>0.5\end{cases}
$$

The phase response parameter $a \in(0,1)$ determines the interaction strength, i.e., the effect an incoming pulse has on an oscillator's phase. The used combination of an excitatory and an inhibitory coupling domain has beneficial effects on synchronization [14,23]. The function is simple enough to be implemented in resource-constraint systems, where it serves as a kind of proportional control mechanism, drawing the receiving oscillators' phases towards zero, i.e., to the current phase of the sending oscillator. The exchange of pulses between coupled oscillators thus reduces the differences between their phases over time. For oscillators numbered in the order of their next firing time, such that $\phi_{k}<\phi_{k-1}$ for $k \in[2 \ldots n]$, we evaluate the level of synchronization in the graph by the phase spread $s:=1-\max _{k} \delta_{k}$, where $\delta_{k}=\left(\phi_{k-1}-\phi_{k}\right)(\bmod 1)$ is the phase gap leading up to the $k$ th oscillator. The phase spread $s$ represents the length (normalized by $2 \pi$ ) of the smallest connected interval on the unit circle that contains all points $\varphi_{k}=\exp \left[2 \pi i \phi_{k}\right]$. As the system synchronizes, the largest phase gap tends to one: $\delta_{\max } \rightarrow 1$. If the phases are degenerate, their order is not well defined. The definition of the phase spread holds for any of the allowed orders. If all phases are equal $\left(\phi_{k}=\phi \forall k\right)$, we speak of a perfectly synchronized system, as all firing events always occur simultaneously. A nonsynchronized system with the phase response function (1) does not reach perfect synchronization in finite time but is considered to be synchronized if $s$ is below a certain threshold.

All leaf nodes receive pulses only from $v_{c}$ but not from the other leaf nodes. Thus, the synchronization process is advanced only by the firing events of $v_{c}$. Upon such an event, all leaf nodes change their phases according to (1), and the phase gaps change to

$$
\delta_{k}^{+}= \begin{cases}\delta_{k}+a\left(1-\delta_{k}\right) & \text { if } 0.5 \in\left[\phi_{k}, \phi_{k-1}\right) \\ (1-a) \delta_{k} & \text { else. }\end{cases}
$$

Illustrating all phases as points on the unit circle, these phase gaps are arcs on the circle (Fig. 1). Always one such arc contains the phase $\phi=0.5$. A pulse from $v_{c}$ lengthens this antipodal arc but shortens all other arcs.

\section{DEADLOCKS}

Since (1) allows for inhibitory effects from receiving pulses, it is conceivable that $v_{c}$ never fires. Such a deadlock

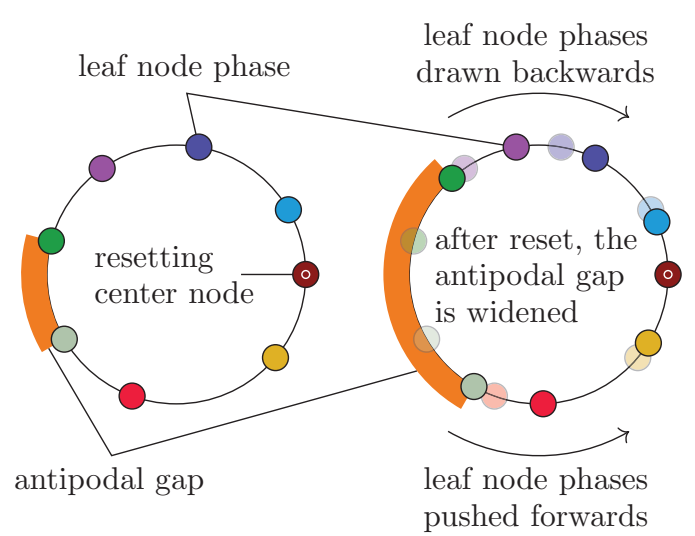

FIG. 1. The center node fires. Phase constellation before the fire event (left) and after reception of the pulse by leaf nodes (right). The antipodal phase gap increases; all other gaps decrease.

occurs if the phase $\phi_{c}\left(t_{k}\right)$ on the verge of receiving a pulse from $v_{k}$ is in the inhibitory domain for all $k \in[1 \ldots n]$. In fact, deadlocks may occur for any phase response function with an inhibitory part, including delay-only functions. A discussion of piecewise linear delay-advance phase response functions with a switching phase $\phi_{s} \neq 0.5$ goes beyond the scope of this work but follows the same train of thoughts. Formally, a deadlock state is a configuration of the phases that prevent at least one oscillator's phase from reaching 1 . There are configurations that lead to a deadlock state only after every oscillator has fired at least once. In simulations, we have regularly seen this transition to a deadlock for random graphs but never for star graphs. Thus, the following analysis of deadlocks in star graphs does not cover the domain of attraction toward the set of these configurations. To gain an understanding of the process that keeps $v_{c}$ in a deadlock, we focus on the sequence of phases that $v_{c}$ exhibits right before the firing events of each of the leaf nodes.

\section{A. Setup}

We randomly initialize the $n$ leaf nodes, which then evolve according to the described model, thus firing periodically, independent from each other at times $t_{k}+m T$ with $k \in[1 \ldots n]$ and $m \in \mathbb{N}$. For simplicity, we label the leaf nodes according to their firing order. The center node's phase $\phi_{c}$ begins to evolve with $\phi_{c}\left(t_{0}\right)=0$. Its phase right before receiving a pulse from a leaf node determines whether it moves forward (excitatory coupling) or backward (inhibitory coupling) as a reaction.

Let $t_{0}$ coincide with the time directly after a firing event of $v_{n}$. If $v_{c}$ is caught in a deadlock, only inhibitory coupling will occur. Thus, in a deadlock, right before $v_{k}$ fires at time $t_{k}$, the center node exhibits the phase

$$
\phi_{c}\left(t_{k}^{-}\right)=\phi_{c}\left(t_{0}\right)(1-a)^{k-1}+\sum_{l=1}^{k} \delta_{l}(1-a)^{k-l} .
$$

If any of these phases is larger than one half, the center node "escapes" the inhibitory domain and will fire at some point in time and consequently not be in a deadlock.

Before determining the domain of initial phase gaps that keep $v_{c}$ in a deadlock, we first derive that in such a deadlock 
the phases in (2) converge monotonically to a distinct, finite sequence $\left(\Phi_{k}\right)_{k \in[1 . . n]}$ dependent only on the initial phases of all leaf nodes $v_{k}$. Then we derive the fraction of initial configurations of leaf node phases that lead to such sequences.

\section{B. Limit behavior of $\phi_{c}$}

For the analysis of convergence one commonly uses the system's return map, i.e., the function that maps an oscillator's current phase to its phase after one period $T=1$ has passed. Since in a deadlock all nodes but $v_{c}$ oscillate undisturbed without incoming pulses, only the return map for $v_{c}$ is of interest. After one period every leaf node fired exactly once. So the phase of $v_{c}$ will have changed from $\phi_{c}\left(t_{0}\right)$ to $(1-a) \phi_{c}\left(t_{n}\right)$ due to its internal dynamics and reaction to received pulses.

We now show that a fixed point $\Phi_{0}$ of this return map exists and that

$$
\left(\xi_{0, m}\right)_{m \in \mathbb{N}}:=\phi_{c}\left(t_{0}+m T\right)
$$

converges monotonically to $\Phi_{0}$. Further, because $\phi_{c}\left(t_{k}\right)$ is fully defined by $\phi_{c}\left(t_{0}\right)$ and the times between consecutive pulses $\delta_{j}$, for $j \in[1 \ldots k], \Phi_{0}$ analogously defines a finite sequence $\left(\Phi_{k}\right)_{k \in[1 . . n]}$ of center node phases before the firing of $v_{k}$. We will show that the sequences

$$
\left(\xi_{k, m}\right)_{m \in \mathbb{N}}:=\phi_{c}\left(t_{k}+m T\right)
$$

likewise converge monotonically to $\Phi_{k}$, respectively.

If a fixed point of the return map exists, it fulfills $\phi_{c}\left(t_{0}\right)=(1-a) \phi_{c}\left(t_{n}\right)$. From this and (2) we derive $\Phi_{0}$, which coincides with $v_{c}$ 's phase after the reception of pulse a from $v_{n}$ in a converged state,

$$
\Phi_{0}=\frac{1-a}{1-(1-a)^{n}} \sum_{l=1}^{n} \delta_{l}(1-a)^{n-l},
$$

with $a \neq 0$. The elements of the converged sequence $\left(\Phi_{k}\right)_{k \in[1 . . n]}$ are then defined by

$$
\Phi_{k}=\Phi_{0}(1-a)^{k-1}+\sum_{l=1}^{k} \delta_{l}(1-a)^{k-l} .
$$

To show convergence, we use the recursive definition of (3),

$$
\xi_{0, m}=\xi_{0, m-1}(1-a)^{n}+G,
$$

with the added constant

$$
G:=(1-a) \sum_{l=1}^{n} \delta_{l}(1-a)^{n-l}
$$

and expand it to an indexed notation

$$
\xi_{0, m}=\xi_{0,0}(1-a)^{m n}+G \sum_{l=0}^{m-1}(1-a)^{l n} .
$$

In the limit of $m \rightarrow \infty$, the sequence converges to

$$
\xi_{0, \infty}=\frac{1}{1-(1-a)^{n}} G=\Phi_{0}
$$

The convergence is monotonic because

$$
\xi_{0, m+1}-\xi_{0, m}=\underbrace{(1-a)^{m n}\left(1-(1-a)^{n}\right)}_{>0}\left(\Phi_{0}-\xi_{0,0}\right),
$$

which is positive (increasing) if $\xi_{0,0}<\Phi_{0}$ and negative (decreasing) if $\xi_{0,0}>\Phi_{0}$.

Since we required $0=\xi_{0,0}<\Phi_{0}$, by comparing (2) and (4) we see that the sequences $\left(\xi_{k, m}\right)_{m \in \mathbb{N}}$ also converge monotonically to their limits $\Phi_{k}$.

\section{Volume of deadlock configurations}

Now that we know the long-term behavior of $v_{c}$, we define a condition for its limit sequence $\Phi_{k}$ that is equivalent to $v_{c}$ being in a deadlock. The map from phase gaps between leaf nodes $\delta_{k}$ to the sequence of center node phases $\Phi_{k}$ is linear and defined by an $n \times n$ matrix $A$ with entries

$$
A_{k m}=\frac{(1-a)^{k-m}}{1-(1-a)^{n}} \times \begin{cases}1 & k \geqslant m \\ (1-a)^{n} & \text { else. }\end{cases}
$$

A deadlock occurs if all center node phases in the sequence $\Phi_{k}$ are smaller than one half. Using the matrix $A$, the deadlock condition is thus given by

$$
0.5 \succeq \boldsymbol{\Phi}=A \delta
$$

with $\boldsymbol{\Phi}=\left(\Phi_{1}, \ldots, \Phi_{n}\right)^{\mathrm{T}}$, the vector consisting of the elements of $\left(\Phi_{k}\right)_{k \in[1 . . n]}$, and $\delta=\left(\delta_{1}, \ldots, \delta_{n}\right)^{\mathrm{T}}$. The symbol $\succeq$ refers to an element-wise $\geqslant$ relation. Note that $A$ depends only on $a$ and is thus fixed for a given phase response function. To determine the domain of deadlock configurations (deadlock domain), we observe that the configuration space (i.e., the space of all combinations of phase gaps $\boldsymbol{\delta}$ ) is the standard $(n-1)$-simplex in $\mathbb{R}^{n}$,

$$
S_{n}=\left\{\delta \in \mathbb{R}^{n} \mid \delta_{k} \geqslant 0 \wedge \sum_{k} \delta_{k}=1\right\} .
$$

The deadlock domain, which is a subset of $S_{n}$, is

$$
D_{n}=\left\{\delta \in S_{n} \mid 0 \preceq A \delta \preceq 0.5\right\} .
$$

To get an idea of the incidence of deadlock occurrences, we derive the fraction of initial configurations exhibiting deadlock behavior over all possible configurations, which corresponds to calculating the $(n-1)$-dimensional volumes of $D_{n}$ and $S_{n}$ embedded in $\mathbb{R}^{n}$. Per definition of the phase gaps we have

$$
\delta_{n}=1-\sum_{m=1}^{n-1} \delta_{m}
$$

which defines a hypersurface in $\mathbb{R}^{n}$, whose $(n-1)$ dimensional volume is given by

$$
v=\int_{V} \sqrt{n} d V
$$

where the factor $\sqrt{n}$ is the Jacobian determinant of the parametrization and $d V$ denotes the multidimensional volume element. For the entire configuration space (i.e., $V=S_{n}$ ) the integral yields

$$
v\left(S_{n}\right)=\int_{0}^{u_{1}} \ldots \int_{0}^{u_{n-1}} \sqrt{n} d \delta_{1} \ldots d \delta_{n-1}=\frac{\sqrt{n}}{(n-1) !}
$$


where $u_{k}$ is defined by the requirements that it may not fall below zero and the sum of all $\delta_{k}$ needs to be one:

$$
u_{k}=\max \left(0,1-\sum_{m=1}^{k-1} \delta_{m}\right) \text {. }
$$

Similarly, the integral over the deadlock region takes the form

$$
v\left(D_{n}\right)=\int_{l_{1}}^{u_{1}} \ldots \int_{l_{n-1}}^{u_{n-1}} \sqrt{n} d \delta_{1} \ldots d \delta_{n-1},
$$

but the upper and lower limits $\left(u_{k}\right.$ and $\left.l_{k}\right)$ need more consideration than for (8). In addition to (7), the limits are governed by (6). Since the upper and lower limits of $\delta_{k}$ are affected by both preceding as well as following $\delta_{m \neq k}$, it is not straightforward to define exact limits for each $\delta_{k}$. We can, however, derive the exact limits for $\delta_{n-1}$ and use the extra condition $l_{n-1} \leqslant u_{n-1}$ to reduce the feasible integration interval $I_{n-1}:=\left[l_{n-1}, u_{n-1}\right]$ to a point (i.e., no contribution to the overall integral) if $\delta_{1}, \ldots, \delta_{n-2}$ allow for at most one value of $\delta_{n-1}$ that has $\boldsymbol{\delta}$ lie in the deadlock domain.

As before, $\delta_{n}$ is determined by (7). We also keep the upper limits from (9) for $k<n$.

The additional limitations on $\delta_{n-1}$ come from (6). Using (7) and the definitions

$$
\begin{aligned}
\tilde{A}_{k m} & :=\left\{\begin{array}{ll}
A_{k m}-A_{k n} & m \neq n \\
A_{k n} & m=n
\end{array} \quad k, m \in[1 \ldots n],\right. \\
\boldsymbol{\delta}^{\prime} & :=\left(\delta_{1}, \ldots, \delta_{n-1}, 1\right)^{\mathrm{T}},
\end{aligned}
$$

we rewrite (6) to form the $n$ conditions

$$
\tilde{\boldsymbol{A}}_{m} \cdot \boldsymbol{\delta}^{\prime} \leqslant \frac{1}{2}, \quad \text { with } m \in[1 \ldots n] .
$$

From (11) we see that the entry $\tilde{A}_{m k}$ is positive if $k \leqslant m<n$ and negative otherwise. Thus, rearranging (12) for $\delta_{n-1}$ and introducing

$$
\Theta_{m}:=\frac{1}{\tilde{A}_{m(n-1)}}\left(\frac{1}{2}-\sum_{\substack{j=1 \\ j \neq n-1}}^{n} \tilde{A}_{m j} \delta_{j}^{\prime}\right), \quad \Theta_{0}:=0
$$

and the index set

$$
M:=[1 \ldots n] \backslash\{n-1\},
$$

we find for $m \in M$ the lower constraints $\delta_{n-1} \geqslant \Theta_{m}$ and for $m=n-1$ the upper constraint $\delta_{n-1} \leqslant \Theta_{n-1}$. Then

$$
l_{n-1}=\max _{m \in M \cup\{0\}} \Theta_{m} .
$$

Depending on $a$, there are $\boldsymbol{\delta}^{\prime}$ for which $\exists m \in M$ s.t. $\Theta_{m}>\Theta_{n-1}$. Such $\delta^{\prime}$ do not represent configurations in the deadlock domain because $\Theta_{n-1} \leqslant \delta_{n-1} \leqslant \Theta_{m \neq n-1}$ follows from the transformed deadlock condition (12) and it contradicts $\Theta_{n-1} \leqslant \Theta_{m}$. For the integration we thus have to require that $u_{n-1} \geqslant l_{n-1}$. So the upper limit is

$$
u_{n-1}=\max \left\{l_{n-1}, \min \left\{1-\sum_{j=1}^{n-2} \delta_{j}, \Theta_{n-1}\right\}\right\} \text {. }
$$

If the bounds of $\delta_{n-1}$ reduce its range to $l_{n-1}=u_{n-1}$, the integral over $\delta_{n-1}$ is zero and thus the entire integral will not

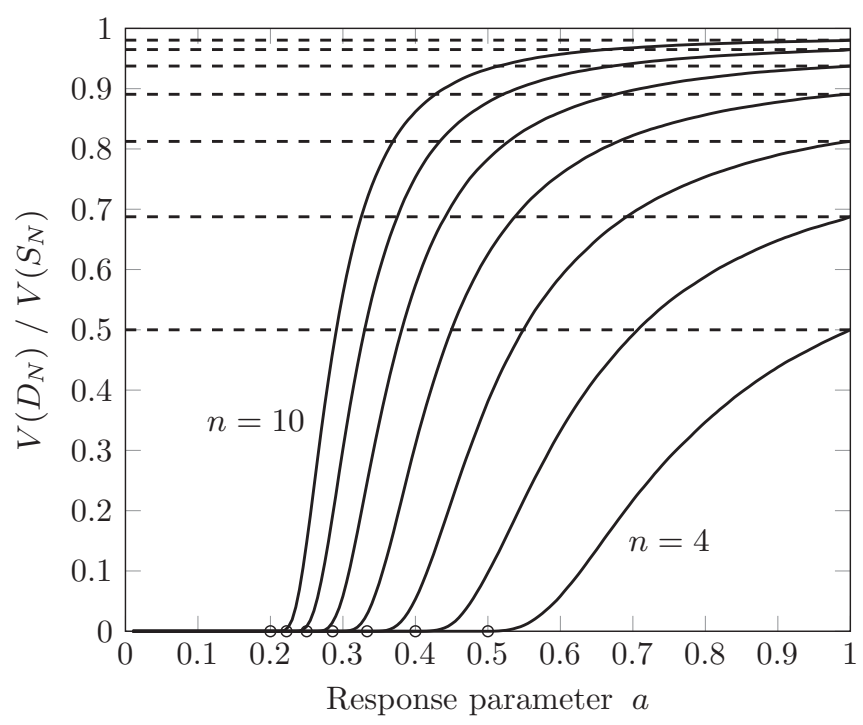

FIG. 2. Fraction of oscillator configurations leading to a deadlock in the synchronization of star graphs. Deadlocks occur only for a sufficiently large response parameter $a>2 / n$ (small circles). They become more frequent with increasing $a$ and number of leaf nodes $n$. Shown are the curves for $n \in\{4, \ldots, 10\}$. For the maximum response $a=1$, the oscillators reset their phases straight to the beginning or end of their cycle. This also holds when all configurations with all $\delta_{k} \leqslant \frac{1}{2}$ (dashed lines) lead to a deadlock (if $n>2$ ).

get contributions from that particular combination $\delta$. Therefore we do not need to further constrain the bounds on $\delta_{k<n-1}$, and we leave them as in (8) and (9).

Solving (10) and dividing the result by (8) gives the fraction of configurations leading to a deadlock. Figure 2 shows this fraction as a function of the response parameter $a$ for different values of the number of leaf nodes $n$.

For many values of $a$, the actual domain of configurations leading to a deadlock is much smaller than the domain of configurations excluded in Refs. [6,16,17]. In fact, no deadlocks occur for $a \leqslant 2 / n$. This in turn means that many more configurations may synchronize than these papers prove. For $a=1$, all configurations with $\delta_{k} \leqslant \frac{1}{2} \forall k$ keep $v_{c}$ in a deadlock. This maximum deadlock domain $\hat{D}_{N}$ is also the complement to the domain of guaranteed synchronization. Its fraction of the entire configuration space is $V\left(\hat{D}_{N}\right) / V\left(S_{N}\right)=1-n / 2^{n-1}$.

\section{RELEVANCE OF DEADLOCKS}

As we derived the incidence of deadlocks in star graphs, the question arises whether this phenomenon plays a role in other graphs as well. Since our analytic approach is tailored to star graphs and in this form works only for them, we now investigate the synchronization behavior numerically, focusing on random graphs, as a prominent example.

Our simulation employs Gilbert random graphs $G(n, p)$ with $n$ nodes and connection probability $p$ [24]. We use five different orders $n=15,20, \ldots, 35$ and generate five random graphs for each of them. For given $n$, the value of $p$ is chosen from a uniform distribution between $2 / n$ and $7 / n$. The response parameter $a$ is varied from 0.05 to 1 . For each graph and response parameter, we initialize the nodes with 


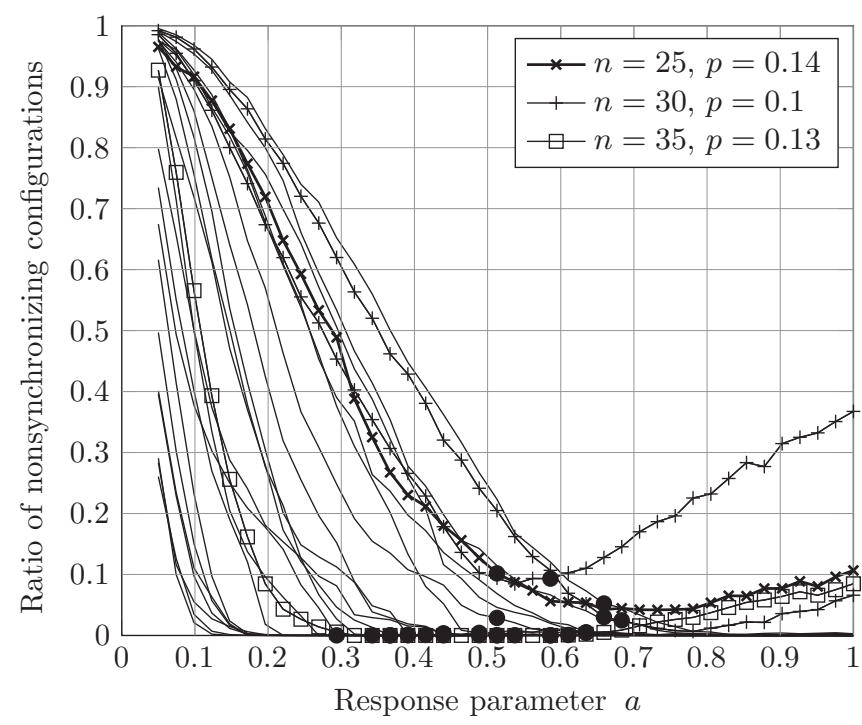

FIG. 3. Ratio of initial configurations that do not lead to synchrony for 25 connected random graphs $G(n, p)$ with $n$ nodes and connection probability $p$ as a function of the response parameter a. There are many nonsynchronizing configurations for weak interactions (small $a$ ), all of which lead to periodic states. For strong interaction (large $a$ ), most configurations lead to synchrony, but the ones that do not synchronize have at least one deadlock node. The $(n, p)$-values of those graphs are given and their curves are marked. For each graph, the dot marks the smallest $a$ for which more than half of the nonsynchronizing configurations exhibit deadlocks.

a random phase between zero and one, simulate the system over 2000 periods, and record the state to which the system has converged. The process is repeated 3000 times for each combination of parameters. The ratio of nonsynchronizing configurations in these runs is shown in Fig. 3.

As a result, we find two types of stable, nonsynchronized states. The first type is a periodic state, where all nodes agree on a common frequency but do not align the start of their cycles, despite regular pulse exchange between all neighbors. These states are responsible for the rise of nonsynchronizing configurations for small values of $a$. The second type is a deadlock state, where at least one node $v_{c}$ never fires and thus also never synchronizes with the rest of the network. States exhibiting deadlocks make up the nonzero ratios of nonsynchronizing configurations for large values of $a$. Two examples of an 11-node random graph with initial configurations leading to a periodic state and a deadlock state, respectively, are shown in Fig. 4.

In our simulations, the probability of periodic, nonsynchronized states to occur is highest for weak interactions and tends to zero or a small value at stronger interactions. In contrast, graphs that exhibit deadlocks have an increased probability of converging to a deadlock state for very strong interactionsfor one of the generated graphs even up to almost $40 \%$.

A node whose phase is reduced due to an incoming pulse effectively has a prolonged time spent in the inhibitory domain. The stronger the coupling, the longer this time becomes, making it more likely to receive another inhibitory pulse. As we have seen for star graphs, with sufficiently many incoming pulses, a node can be kept in a deadlock and stronger

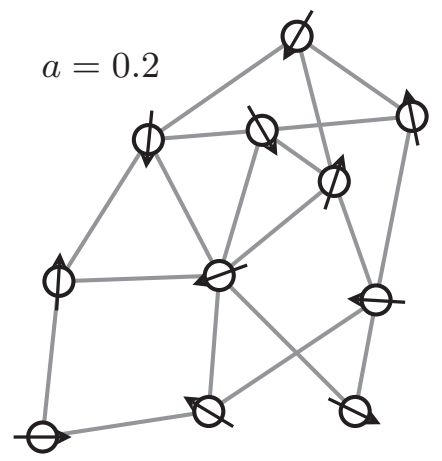

(a)

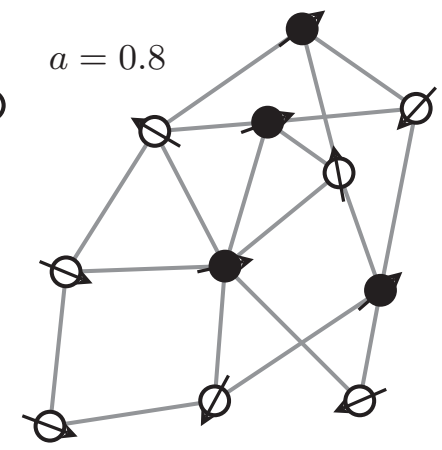

(b)

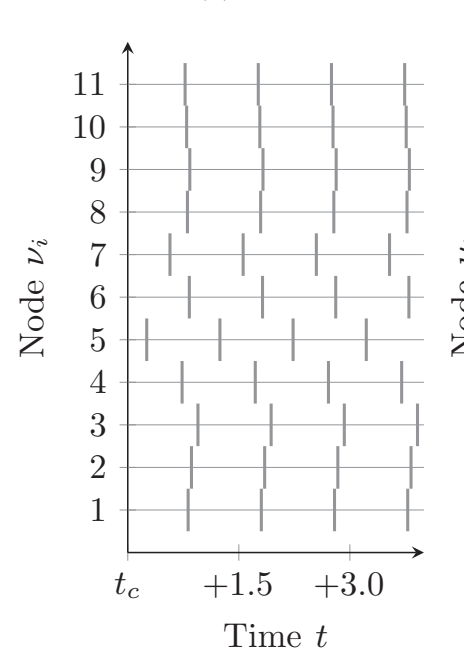

(c)

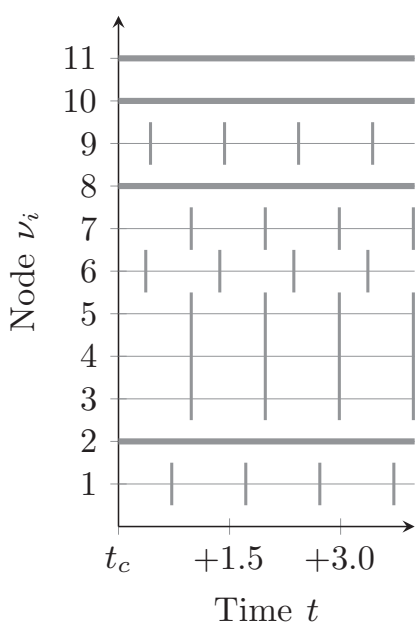

(d)
FIG. 4. Examples of nonsynchronizing configurations. Upper row: graph and two sets of initial phase configurations, indicated by the directions of the arrows. Lower row: pulses over a few periods after a sufficient convergence time. Left side: a periodic state that does not further synchronize despite repeated communication. Right side: some nodes in a deadlocked state [marked black in (b)], in which they are prevented from firing; the remaining nodes have converged to a periodic state.

interaction reduces the number of pulses required for the deadlock, i.e., makes the deadlock more likely to occur in a random graph.

In star graphs we did not encounter nonsynchronized periodic states without deadlocks. The effects allowing a network of pulse-coupled oscillators to converge to a periodic state have been studied [19], but a detailed discussion goes beyond the scope of our paper.

Deadlock states appear in two situations, with different impact on the synchronization. First, they may appear as a side effect of a periodic state. If $v_{c}$ gets enough inhibitory input from its neighbors in the periodic state, it never fires, but its removal from the network would have no impact on the system. Second, $v_{c}$ is a bottleneck in the graph, i.e., removing $v_{c}$ from the graph splits it into more than one component. As a bottleneck, the firing behavior of $v_{c}$ directly impacts the synchronization of the remaining nodes, since no information is transmitted from one of the otherwise disconnected components to another, without $v_{c}$ firing. 
In some systems, completing the internal cycle of a node is necessary for other internal processes, for instance, to facilitate the transfer of information to neighbors. Thus, preventing a node from firing may have more severe consequences than a nonsynchronized, periodic message exchange. A deadlock state is thus qualitatively different from other nonsynchronized states, and its potential presence in random graphs makes an analysis of the phenomenon a worthwhile endeavor.

\section{CONCLUSIONS}

This paper analyzed a deadlock effect that prevents certain graphs of pulse-coupled oscillators from synchronizing. For star graphs, we showed that the domain of configurations exhibiting a deadlock is, for some intervals of the response parameter $a$, only a small subset of the configurations that are excluded in convergence proofs found in the literature. This result provides an analytical upper bound for the volume of synchronizing states, complementing the existing lower bound $[6,16]$. We computed the fraction of initial states leading to a deadlock in star graphs and found a condition for the maximum steepness of the phase response function necessary for exhibiting deadlocks. Simulations show that synchronization deadlocks can also occur in random graphs. In both star and random graphs, deadlocks seem to be more prominent if there is a strong reaction to incoming pulses and require the affected nodes to have a certain minimum degree. Knowing this phenomenon complements our understanding of nonsynchronizing behavior and could help in the design of network synchronization techniques.

\section{ACKNOWLEDGMENT}

This work was partly funded by the Austrian Science Fund (FWF), grant "Self-organizing synchronization with stochastic coupling" (P30012).
[1] C. Peskin, Mathematical Aspects of Heart Physiology, Courant Institute Lecture Notes (Courant Institute of Mathematical Sciences, New York University, New York, 1975).

[2] A. T. Winfree, J. Theor. Biol. 16, 15 (1967).

[3] E. Brown, J. Moehlis, and P. Holmes, Neural Comput. 16, 673 (2004).

[4] A. F. Meyer, R. S. Williamson, J. F. Linden, and M. Sahani, Front. Syst. Neurosci. 10, 109 (2017).

[5] R. Mathar and J. Mattfeldt, SIAM J. Appl. Math. 56, 1094 (1996).

[6] J. Klinglmayr, C. Kirst, C. Bettstetter, and M. Timme, New J. Phys. 14, 073031 (2012).

[7] Y. Wang, F. Nunez, and F. J. Doyle, IEEE Trans. Control Syst. Tech. 21, 1455 (2013).

[8] R. E. Mirollo and S. H. Strogatz, SIAM J. Appl. Math. 50, 1645 (1990).

[9] P. Bressloff and S. Coombes, Physica D 126, 99 (1999).

[10] C. Van Vreeswijk, L. F. Abbott, and G. B. Ermentrout, J. Comput. Neurosci. 1, 313 (1994).

[11] Á. Corral, C. J. Pérez, A. Díaz-Guilera, and A. Arenas, Phys. Rev. Lett. 75, 3697 (1995).
[12] C. v. Vreeswijk and H. Sompolinsky, Science 274, 1724 (1996).

[13] M. Rhouma and H. Frigui, IEEE Trans. Pattern Anal. Machine Intell. 23, 180 (2001).

[14] A. Abouzeid and B. Ermentrout, Phys. Rev. E 80, 011911 (2009).

[15] C. C. Canavier and R. A. Tikidji-Hamburyan, Phys. Rev. E 95, 032215 (2017).

[16] H. Gao and Y. Wang, Automatica 104, 196 (2019).

[17] F. Núñez, Y. Wang, and F. J. Doyle, Automatica 52, 202 (2015).

[18] J. Nishimura and E. J. Friedman, Phys. Rev. E 86, 025201(R) (2012).

[19] C. C. Canavier and S. Achuthan, Math. Biosci. 226, 77 (2010)

[20] D. J. Watts and S. H. Strogatz, Nature 393, 440 (1998).

[21] G. A. Pagani and M. Aiello, Physica A 392, 2688 (2013).

[22] A. Clauset, C. R. Shalizi, and M. E. J. Newman, SIAM Rev. 51, 661 (2009).

[23] J. Nishimura and E. J. Friedman, Phys. Rev. Lett. 106, 194101 (2011).

[24] E. N. Gilbert, Ann. Math. Stat. 30, 1141 (1959). 